\title{
The direct boundary element method: 2D site effects assessment on laterally varying layered media (methodology)
}

\author{
Sonia Álvarez-Rubio ${ }^{\mathrm{a}}$, Francisco J. Sánchez-Sesma ${ }^{\mathrm{b}}$, Juan José Benito ${ }^{\mathrm{c}}$, Enrique Alarcón ${ }^{\mathrm{d}}$ \\ aDepartamento de Matemática Aplicada, E.U.I.T. Industrial, Universidad Politécnica de Madrid, Ronda de Valencia, 3, 28012 Madrid, Spain \\ 'Instituto de Ingeniería, UNAM, Cd. Universitaria, Apdo. 70-472, Coyoacán 04510 México D.F., Mexico \\ ${ }^{\circ}$ Departamento de Ing. de Construcción y Fabricación, E.T.S.I. Industriales, Universidad Nacional Distancia, Apdo. 60.149, 28080 Madrid, Spain \\ ¿Departamento de Mecánica Estructural y Construcciones Industriales, E.T.S.I. Industriales, \\ Universidad Politécnica de Madrid, José Gutiérrez Abascal, 2, 28006 Madrid, Spain
}

\begin{abstract}
The Direct Boundary Element Method (DBEM) is presented to solve the elastodynamic field equations in 2D, and a complete comprehensive implementation is given. The DBEM is a useful approach to obtain reliable numerical estimates of site effects on seismic ground motion due to irregular geological configurations, both of layering and topography.

The method is based on the discretization of the classical Somigliana's elastodynamic representation equation which stems from the reciprocity theorem. This equation is given in terms of the Green's function which is the full-space harmonic steady-state fundamental solution. The formulation permits the treatment of viscoelastic media, therefore site models with intrinsic attenuation can be examined. By means of this approach, the calculation of 2D scattering of seismic waves, due to the incidence of $\mathrm{P}$ and $\mathrm{SV}$ waves on irregular topographical profiles is performed. Sites such as, canyons, mountains and valleys in irregular multilayered media are computed to test the technique. The obtained transfer functions show excellent agreement with already published results.
\end{abstract}

Keywords: 2D site effects; Irregular multilayered medium; Direct boundary element method

\section{Introduction}

Recent destructive earthquakes have shown significant evidences of the effects of surface geology and topography on ground motion characteristics at a given site. Among these effects we have: amplification and deamplification patterns, strong spatial variability of amplification and polarization within a small area [1-6].

There are several ways of assessing site effects. The characterization of a given site can be achieved based on instrumental or theoretical-analytical or numericalapproaches to the problem. A detailed survey of the numerical methods developed for the computation of site effects is given by Sánchez-Sesma [7]. Among the various techniques, the Boundary Integral Equation Method (BIEM) has gone through significant development and offers some advantages. There are two main approaches: (1) in the direct formulation (DBIEM) one finds the unknown tractions and displacements at the boundaries of the domains, while (2) in the indirect approach (IBIEM) one formulates the problem in terms of force or moment boundary densities. At this point there is a terminology issue we want to address: because the DBIEM parallels in many aspects the Finite Element Method (FEM), the term BEM (for Boundary Element Method) was coined naturally in the 1970 s by Brebbia [8] and is used extensively since then. Therefore, the DBEM (or simply BEM which is usually referred to as the standard) and the IBEM will stand in this work for the direct and the indirect formulations of BEM, respectively.

Some significant studies carried out using an indirect approach were presented by Luco et al. [9] and Luco and de Barros [10] in which a 2.5D indirect formulation was proposed in order to obtain the 3D response of an infinitely long canyon, and a class of cylindrical inclusions embedded in layered media, respectively. In Sánchez-Sesma and Campillo [11,12], the Indirect Boundary Element Method 
(IBEM) was presented and used to compute the 2D seismic response of topographical irregularities on the surface of a homogeneous half-space. Pedersen et al. [13] used the IBEM to compute the 3D response of $2 \mathrm{D}$ topographies, while Sánchez-Sesma and Luzón [14] and Luzón et al. [15] applied the method to study 3D alluvial valleys and topographies. Therefore, the indirect approaches have been widely used for this topic.

On the other hand, several studies have been carried out using the direct formulation. For instance, Kawase [16] proposed a discrete wavenumber boundary integral equation method for 2D scattering problems. This approach has been extended to $3 \mathrm{D}$ problems by $\mathrm{Kim}$ and Papageorgiou [17] and to 2.5D elastodynamic scattering problems by Papageorgiou and Pei [18]. The Direct Boundary Element Method (DBEM) using the full-space Green's function allowed Reinoso $[19,20]$ to consider the $2 \mathrm{D}$ and $3 \mathrm{D}$ seismic response on homogeneous alluvial settings.

This work is focused on the seismic response of $2 \mathrm{D}$ irregular topographies (both underground and superficial, that shape laterally varying media) under incoming waves. For this purpose a DBEM formulation of the problem has been developed. The main site categories which are related to important local topographical and geological effects on seismic ground motion, are addressed in this formulation, which stems from the classical weighted residual technique; the approach can be regarded as an extension to elastodynamics of Betti's classical reciprocal theorem of elastostatics. Moreover, it uses the infinite-space 2D harmonic Green's function [8,21]. Therefore, the method is based upon Somigliana's elastodynamic equation. The simulations have been carried out in the frequency-domain.

Our approach to these complex site effects issues, is based on the development of DBEM formulation for the treatment of the two different situations that can take place: the internal and the external problems. Hence, the computations are carried out in terms of total and diffracted fields, respectively. Of course, the boundary conditions on each irregular interface between layers are explicitly enforced in our approach. The formulation has been systematically implemented on a versatile computer code in which the different irregularly shaped layers of the studied domain can be considered. This implementation of the DBEM is restricted to the in-plane $(\mathrm{P}-$ $\mathrm{SV})$ problem under incidence of elastic plane waves. The antiplane ( $\mathrm{SH}$ ) case will be discussed elsewhere.

As a first test the DBEM results are compared with those obtained from the application of the IBEM as presented in Sánchez-Sesma and Campillo [11,12]. The verification is performed for simple theoretical models of topographical reliefs. It is confirmed that these profiles may induce significant variations on earthquake ground motion. The IBEM is based on a single-layer boundary integral representation of the diffracted elastic wave field. It can be shown that this representation can also be derived from Somigliana's identity [11], which is also the departure for the DBEM. Therefore, although the two techniques are closely related, they approach the site effect problem from different points of view. In the case of the IBEM, the computations carried out can be regarded as a numerical realization of Huygens' principle, which states that every point on a wavefront acts as a source. On the other hand, the DBEM can be interpreted as a direct consequence of the virtual work theorem, it implies relationships of boundary values of both displacements and tractions from an energy viewpoint.

A further testing of the method is performed by the comparison between results obtained from the application of the DBEM, and the hybrid method presented by Faccioli et al. [22] to simulate the response of a real site (a multilayered sedimentary basin). While with the DBEM the appropriate matrices are generated for each irregular layer and then assembled, the hybrid pseudospectral approach consists in splitting up the computational domain into two disjoint parts, and using the FEM and the Spectral Element Method (SEM) on either part. The agreement between results (transfer functions at specific sites) is good. However, a detailed analysis of the relative performances of these techniques is beyond the scope of this work.

As part of the DBEM's methodology verification, the grid optimization has been accomplished by comparison of the results derived from different geometric discretization criteria. The investigation on the existence of nonphysical waves (the so called edge effects) has also being part of the validation process. Taking into account all the results achieved by the different tests it can be said that, the DBEM is a reliable and powerful tool for simulating ground motion on irregular multilayered sites. In fact, the said validation constitutes a consistency test of the DBEM. The application of this technique, to a deeper analysis of the seismic response of complex real sites, is being approached. Its contribution to the study of seismic site effect, together with an optimization of the computation process [23-25] will be addressed in subsequent works.

\section{The direct boundary element method}

\subsection{Integral formulation for time-harmonic problems}

The DBEM is a numerical technique based on Somigliana's integral representation theorem in elastodynamics (this reciprocal theorem is an extension of Betti's classical result in static elasticity). In this work the DBEM formulation is presented for time-harmonic problems. There are several advantages of the formulation in the frequency-domain. For instance: (1) the governing equations are reduced as they have less derivatives, (2) the integral representations are simplified, (3) realistic material damping can be introduced in a simple way, and (4) the time-domain response for an arbitrary excitation can be computed from a suit of frequency-domain responses; this is characteristic of linear systems. 
Consider two elastodynamic states defined over a region $D$ (with $\partial D$ as its boundary). The displacements, tractions and body forces for the first state are denoted by $u_{i}, T_{i}$ and $f_{i}$ while for the second one we have $u_{i}^{\prime}, T_{i}^{\prime}$, and $f_{i}^{\prime}$, respectively. The subindex $i$ stands for the in-plane components $(i=1$ and 2 for directions $x_{1}=x$ and $x_{2}=z$, respectively). If both states are time-harmonic with the same frequency $\omega$ and the initial condition values are the same as the steady-state values, the reciprocal theorem [21] equals the work done by body forces and boundary tractions associated to each state over the displacements of the other one. Here and hereafter use is made of the summation convention, i.e. the repetition of a subindex should be understood as a summation over the range of such index.

In case the second state is the full-space time-harmonic fundamental solution or Green's function, that is to say, the response of an infinite elastic medium to a harmonic inplane unit line load of frequency $\omega$, in absence of body forces, the reciprocal theorem becomes Somigliana's integral representation in elastodynamics [21]

$$
\begin{gathered}
C_{j i}(\mathbf{x}, \omega) u_{i}(\mathbf{x}, \omega)+\int_{\partial D} T_{j i}^{\prime}(\mathbf{x}, \mathbf{y}, \omega) u_{i}(\mathbf{y}, \omega) \mathrm{d} S(\mathbf{y}) \\
=\int_{\partial D} U_{j i}^{\prime}(\mathbf{x}, \mathbf{y}, \omega) t_{i}(\mathbf{y}, \omega) \mathrm{d} S(\mathbf{y}),
\end{gathered}
$$

where $U_{j i}^{\prime}(\mathbf{x}, \mathbf{y}, \omega)=G_{i j}(\mathbf{y}, \mathbf{x}, \omega) \equiv$ displacement at point $\mathbf{y}$ in the direction $i$ due to a unit in-plane line load applied at point $\mathbf{x}$ in direction $j, T_{i j}^{\prime}(\mathbf{x}, \mathbf{y}, \omega) \equiv i$ th component of traction at boundary point $\mathbf{y}$ (at $\partial D$, with normal assumed to point outside $D$ ) due to a unit in-plane line load at point $\mathbf{x}$ in direction $j, \mathbf{x} \in D, \mathbf{y} \in \partial D$, and $i, j=1,2$. The left-hand side integral is understood in the Cauchy's Principal Value sense.

It can be shown that the $k$ th component of both displacements, $u_{k}^{\prime}$, and tractions, $t_{k}^{\prime}$, in $2 \mathrm{D}$ for a concentrated line load along a direction defined by a unit vector $\mathbf{e}$ with components $e_{l}$, can be written in terms of the corresponding Green's functions, or fundamental solutions for a concentrated harmonic load, by means of

$u_{k}^{\prime}(\mathbf{x}, \omega)=U_{l k}^{\prime}(\mathbf{y}, \mathbf{x}, \omega) e_{l}(\mathbf{y})$

and

$t_{k}^{\prime}(\mathbf{x}, \omega)=T_{l k}^{\prime}(\mathbf{y}, \mathbf{x}, \omega) e_{l}(\mathbf{y})$.

The fundamental solutions given by Domínguez [21] in terms of the modified Bessel function of the second kind, are entirely equivalent to the ones that appear in [11] which are written in terms of Hankel's functions.

\subsection{The direct boundary element method}

The analytical solution of the integral equation (1) cannot be obtained in general. Here we present its numerical solution through the application of the DBEM. The first stage consists on the boundary's discretization of the site model analysed. It is divided into $K$ constant elements with

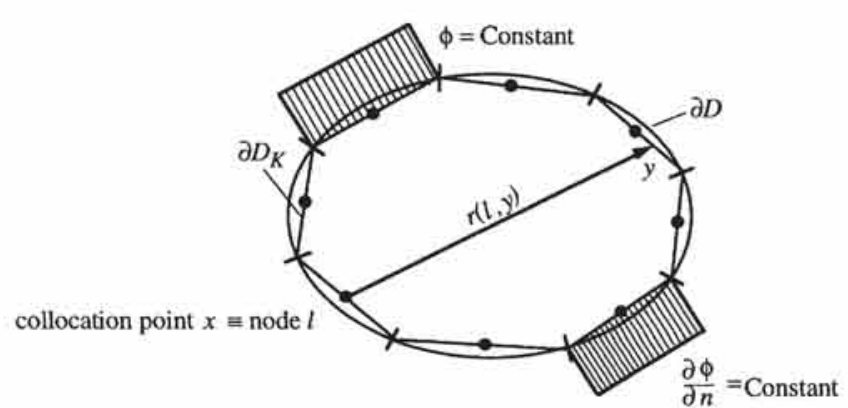

Fig. 1. General scheme of the discretization process of the boundary using constant elements. $\phi$ represents a generic variable field [34].

the nodes at their centers (Fig. 1). That is to say, the unknown values (displacements and tractions) are assumed to be constant over each element.

If such approach is used, the integral equation (1) in case of smooth boundary becomes:

$$
\begin{aligned}
& \frac{1}{2} \delta_{j i} u_{i}(l, \omega)+\sum_{k=1}^{K} u_{i}(k, \omega) \int_{\partial D_{k}} T_{j i}^{\prime}(l, y, \omega) \mathrm{d} s(y) \\
& =\sum_{k=1}^{K} t_{i}(k, \omega) \int_{\partial D_{k}} U_{j i}^{\prime}(l, y, \omega) \mathrm{d} s(y), \quad y \in \partial D_{k} .
\end{aligned}
$$

The collocation point or node where the integral equation is applied is denoted by $l$, while $k$ represents a general element $\partial D_{k}$ that contains the $k$ node (Fig. 1). This expression represents two equations associated to the $j$ axis that can be written

$$
\sum_{k=1}^{K} H_{j i}(l, k) u_{i}(k)=\sum_{k=1}^{K} G_{j i}(l, k) t_{i}(k),
$$

where $H_{j i}$ and $G_{j i}$ are the associated integral coefficients to the displacement and traction vectors, respectively, along the $i$ axis at node $k, u_{i}(k), t_{i}(k)$.

Repeating Eq. (5) for every node, a set of algebraic equations of the type

$$
\underset{(2 K \times 2 K)}{H} \underset{(2 K \times 1)}{\mathbf{u}}=\underset{(2 K \times 2 K)}{G} \underset{(2 K \times 1)}{\mathbf{t}},
$$

can be established. The $2 K \times 2 K$ matrix $H$ and $G$ (where $K$ is the total number of nodes or elements on the boundary), contains the integral coefficients $H_{j i}$ and $G_{j i}$. Whereas the vectors $\mathbf{u}$ and $\mathbf{t}$ correspond to the displacement and tractions values at each of the $K$ nodes.

Two cases must be discussed when the integral coefficients contained in the $H$ and $G$ matrices are calculated. These are computed using the Gauss quadrature integration formulae, when node $l$ does not belong to the element $k$ where the integration is calculated $(l \neq k)$. When the collocation point is the node of the integration element $(l=k)$, the singularity of the fundamental solution requires an analytical integration [21].

The application of the boundary conditions, permits the rearrangement of Eq. (6), leading to a final linear system of 
$2 K$ equations:

$\underset{(2 K \times 2 K)}{A} \underset{(2 K \times 1)}{\mathbf{x}}=\underset{(2 K \times 1)}{\mathbf{F}}$.

The vector $\mathbf{x}$ represents all unknowns-displacements or tractions-within the problem. By solving this last system of equations all the boundary values can be calculated.

The formulation developed is valid for time-harmonic viscoelastic problems where traction and strain are linearly related. In such cases it has only to be taken into account that Lamé constants are complex valued [21].

\section{DBEM applied to site effect estimation}

\subsection{Diffraction of elastic waves by local conditions}

Consider a site defined by a complex local geology and an irregular free surface, such as the one depicted on Fig. 2. The ground motion consists on the superposition of the primary and diffracted waves. The primary waves, also called the free-field, are the solution in the absence of irregularitiesthat is, the waves inciding on the rock basement-whereas the diffracted waves are generated by the interference between the primary waves and the irregularities. Therefore, the total wave field, written in terms of total displacements $\left(\mathbf{u}^{t}\right)$ and total tractions $\left(\mathbf{t}^{\mathrm{t}}\right)$, is

$\mathbf{u}^{\mathrm{t}}=\mathbf{u}^{0}+\mathbf{u}^{\mathrm{d}}$,

$\mathbf{t}^{\mathrm{t}}=\mathbf{t}^{0}+\mathbf{t}^{\mathrm{d}}$

where $\left(\mathbf{u}^{0}, \mathbf{t}^{0}\right)$ denotes the free-field and $\left(\mathbf{u}^{\mathrm{d}}, \mathbf{t}^{\mathrm{d}}\right)$ contains the diffracted waves at the irregularities.

The free-field is analytically known and it is the solution of the transmission and reflection of the in-plane problem ( $\mathrm{P}-\mathrm{SV}$ waves) at the free surface and boundaries of the homogeneous half-space $(z \leq 0)$, see [26].

As it is shown in Fig. 2, the formulation has being developed to deal with multiregions defined by irregular interfaces. In such situations, the solution of the dynamic problem is established by the fulfillment of expression (8) together with the compatibility and equilibrium conditions (continuity of displacements and tractions, respectively), along interfaces. Namely, for any $(i, i+1)$ pair of subregions:

$\mathbf{u}_{i}^{\mathrm{t}}=\mathbf{u}_{i+1}^{\mathrm{t}}$,

$\mathbf{t}_{i}^{\mathrm{t}}=-\mathbf{t}_{i+1}^{\mathrm{t}}$.

In all situations the DBEM is based on the resolution of the Eq. (6). The way to apply it depends on the local conditions. If the site considered requires the modelling of an internal region, the radiation condition of the actual problem is satisfied. In such cases the DBEM is established in terms of the total wave field, $\mathbf{u}^{\mathrm{t}}, \mathbf{t}^{\mathrm{t}},[26]$ :

$H \mathbf{u}^{\mathrm{t}}=G \mathbf{t}^{\mathrm{t}}$.

When the computation involves an external problem, it has to be transformed because the problem itself does not satisfy the radiation condition. The DBEM's implementation is done in terms of the diffracted field, which should satisfy the radiation condition, leading to [26]:

$H \mathbf{u}^{\mathrm{d}}=G \mathbf{t}^{\mathrm{d}}$,

$H\left(\mathbf{u}^{\mathrm{t}}-\mathbf{u}^{0}\right)=G\left(\mathbf{t}^{\mathrm{t}}-\mathbf{t}^{0}\right)$.

Considering the various aspects discussed herein, the formulation presented will be applied to study models that represent geological and topographical conditions of practical interest in seismic site response estimation.

\subsection{Computation of site effects on multilayered media shaped by irregular interfaces}

In general, a realistic DBEM's computation of site effects-up to what the geotechnical, geological and topographical information gathered allows-has to be

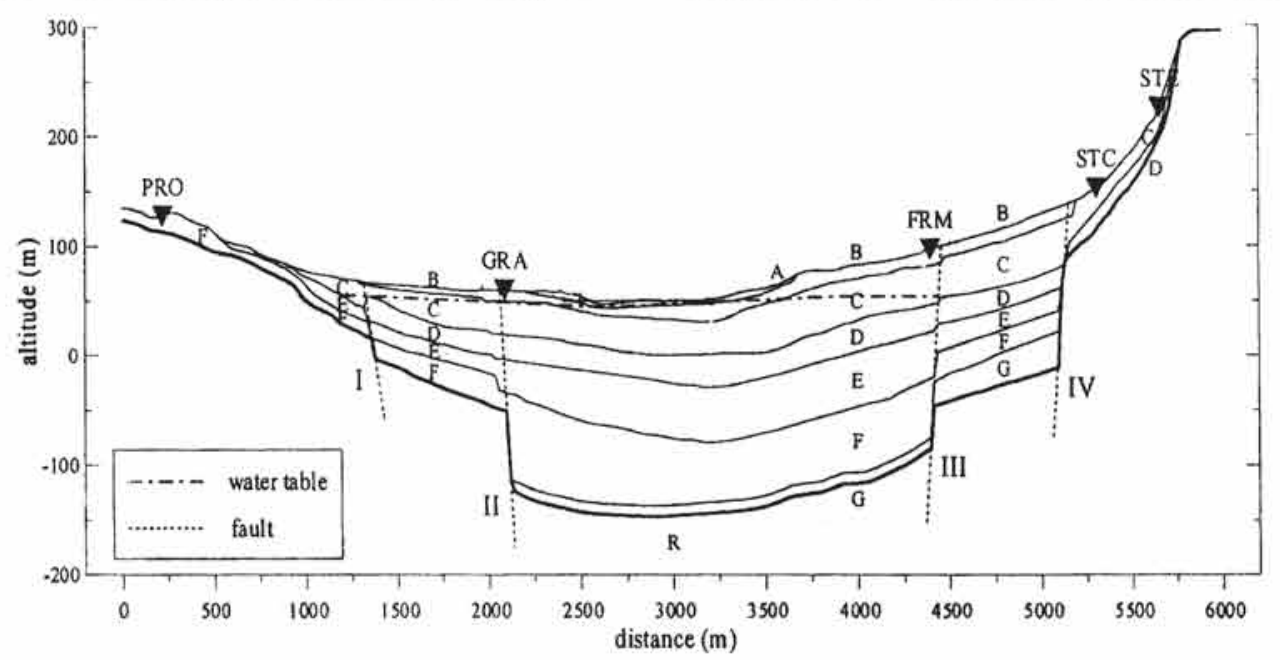

Fig. 2. An example of a multilayered region defined by irregular sub and superficial interfaces. The structure corresponds to a cross-section of the Volvi sedimentary basin close to the city of Thessaloniki in Greece [22]. This basin's structure is referred in this work as model I. 


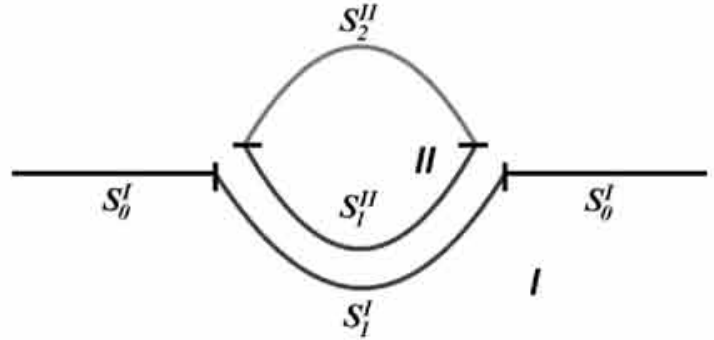

Fig. 3. In the figure is illustrated a scheme of a theoretical site model corresponding to an irregular elevation (medium II) embedded in a homogeneous half-space (medium I). From the point of view of the computation of site effect by the DBEM, medium I corresponds to the external problem, whereas medium II to the internal one.

approached taking into account the formulation of the internal and external problems presented in Section 3.1.

An example of a complex site is shown on Fig. 2. The application of the DBEM to this multilayered irregular valley, which corresponds to a significant type of site on local effects study, consists on the assembly of the solutions arisen from the posing of: the external problem-the total wave field radiated through the homogeneous semi-infinite space which is set as the rock basement (layer $R$ ) - and the internal problems associated to each of the layers of the medium (layers $A-G$ ). The assemblence of both problems will be done through the establishment of the compatibility and equilibrium conditions on the interfaces between layers. In the following sections, the formulation of the internal and external problems will be presented on a more extensive way. The development of it will be based on the theoretical model depicted on Fig. 3. It consists of an elevation represented by medium II (internal problem), embedded on medium I: a homogeneous semi-infinite medium (external problem).

\subsubsection{External problem}

Consider the irregular free surface $S^{\mathrm{I}}\left(S^{\mathrm{I}}=S_{0}^{\mathrm{I}} \cup S_{1}^{\mathrm{I}}\right)$ of the homogeneous half-space $I$ depicted on Fig. 3. $S_{0}^{1}$ corresponds to the flat part of the free surface of the semiinfinite space, whereas $S_{1}^{\mathrm{L}}$ defines the irregular part of the relief of medium I in contact with medium II. The computation of the total motion on the sites along $S_{0}^{\mathrm{I}}$ and $S_{1}^{\mathrm{I}}$ denoted by $\mathbf{u}_{S^{1}}^{\mathrm{t}}, \mathbf{u}_{S^{\mathrm{I}}}^{\mathrm{t}}$, respectively, is based on Eq. (11). Therefore, the implementation of the DBEM leads to the next expanded matrix formulation:

$$
\begin{aligned}
& \left(\begin{array}{ll}
H_{S_{0}^{\mathrm{I}} S_{0}^{\mathrm{I}}} & H_{S_{0}^{\mathrm{I}} S_{1}^{\mathrm{I}}} \\
H_{S_{1}^{\mathrm{I}} S_{0}^{\mathrm{I}}} & H_{S_{1}^{\mathrm{I}} S_{1}^{\mathrm{I}}}^{\mathrm{I}}
\end{array}\right)\left(\begin{array}{c}
\mathbf{u}_{S_{0}^{\mathrm{I}}}^{\mathrm{t}} \\
\mathbf{u}_{S_{1}^{\mathrm{I}}}^{\mathrm{t}}
\end{array}\right) \\
& =\left(\begin{array}{ll}
H_{S_{0}^{\mathrm{I}} S_{0}^{\mathrm{I}}} & H_{S_{0}^{\mathrm{I}} S_{1}^{\mathrm{I}}} \\
H_{S_{1}^{\mathrm{I}} S_{0}^{\mathrm{I}}} & H_{S_{1}^{\mathrm{I}} S_{1}^{\mathrm{I}}}^{\mathrm{I}}
\end{array}\right)\left(\begin{array}{c}
\mathbf{u}_{S_{0}^{\mathrm{I}}}^{0} \\
\mathbf{u}_{S_{1}^{\mathrm{I}}}^{0}
\end{array}\right)+\left(\begin{array}{ll}
G_{S_{0}^{\mathrm{I}} S_{0}^{\mathrm{I}}} & G_{S_{0}^{\mathrm{I}} S_{1}^{\mathrm{I}}} \\
G_{S_{1}^{\mathrm{I}} S_{0}^{\mathrm{I}}} & G_{S_{1}^{\mathrm{S}} S_{1}^{\mathrm{I}}}^{\mathrm{I}}
\end{array}\right) \\
& \times\left(\left(\begin{array}{c}
\mathbf{t}_{S_{0}^{\mathrm{I}}}^{\mathrm{I}} \\
\mathbf{t}_{S_{1}^{\mathrm{t}}}^{\mathrm{t}}
\end{array}\right)-\left(\begin{array}{c}
\mathbf{t}_{S_{0}^{\mathrm{I}}}^{0} \\
\mathbf{t}_{S_{1}^{\mathrm{I}}}^{0}
\end{array}\right)\right) .
\end{aligned}
$$

The displacements, $\mathbf{u}_{S_{i}^{\mathrm{i}}}^{0} i=0,1$, and tractions, $\mathbf{t}_{S^{\mathrm{i}}}^{0} i=0,1$, are the values of the free-field motion of the homogeneous half-space which, as it has been mentioned, are analytically known, and $\mathbf{t}_{S^{\mathrm{t}}}^{\mathrm{t}} i=0,1$ are the known boundary conditions. The unknown of the system are the total displacements across the whole border $S^{\mathrm{l}}, \mathbf{u}_{S^{\mathrm{I}}}^{\mathrm{t}} i=0,1$.

\subsubsection{Internal problem}

The internal problem is represented on Fig. 3 by medium II. It is delimited by its closed boundary $S^{\Pi},\left(S^{\Pi}=S_{1}^{\Pi} \cup S_{2}^{\Pi}\right)$. $S_{2}^{\text {II }}$ is the irregular part of the site's free surface. $S_{1}^{\mathrm{II}}$ coincides with $S_{1}^{\mathrm{I}}$ but they are associated to different media and hence circulated in opposite senses.

According to Eq. (10) the application of the DBEM, in this case, is carried out in terms of the total internal field $\mathbf{u}_{S_{1}^{\mathrm{i}}}^{\mathrm{t}}$, $\mathbf{u}_{S_{2}}^{\mathrm{t}}$. Thus the posing of the problem is:

$$
\left(\begin{array}{ll}
H_{S_{1}^{\mathrm{n}} S_{1}^{\mathrm{n}}} & H_{S_{1}^{\mathrm{n}} S_{2}^{\mathrm{n}}} \\
H_{S_{2}^{\mathrm{n}} S_{1}^{\mathrm{n}}} & H_{S_{2}^{\mathrm{n}} S_{2}^{\mathrm{n}}}
\end{array}\right)\left(\begin{array}{c}
\mathbf{u}_{S_{1}^{\mathrm{n}}}^{\mathrm{t}} \\
\mathbf{u}_{S_{2}^{\mathrm{I}}}^{\mathrm{t}}
\end{array}\right)=\left(\begin{array}{cc}
G_{S_{1}^{\mathrm{n}} S_{1}^{\mathrm{n}}} & G_{S_{1}^{\mathrm{n}} S_{2}^{\mathrm{n}}} \\
G_{S_{2}^{\mathrm{n}} S_{1}^{\mathrm{n}}} & G_{S_{2}^{\mathrm{n}} S_{2}^{\mathrm{n}}}
\end{array}\right)\left(\begin{array}{c}
\mathbf{t}_{S_{1}^{\mathrm{I}}}^{\mathrm{t}} \\
\mathbf{t}_{S_{2}^{\mathrm{n}}}^{\mathrm{t}}
\end{array}\right) .
$$

For the resolution process of this system, the internal and external problems (medium I and II) are assembled by the enforcement of the compatibility and equilibrium conditions at the interface $\left(S_{1}^{\mathrm{I}}, S_{1}^{\mathrm{II}}\right)$. That is:

$\mathbf{u}_{S_{1}^{\mathrm{I}}}^{\mathrm{t}}=\mathbf{u}_{S_{1}^{\mathrm{II}}}^{\mathrm{t}}$,

$\mathbf{t}_{S_{1}^{\mathrm{I}}}^{\mathrm{t}}=-\mathbf{t}_{S_{1}^{\mathrm{II}}}^{\mathrm{t}}$.

\subsubsection{Generalization to a multilayered medium}

The DBEM's application process to site effects estimation explained on previous sections can be generalized to a multilayered site, e.g. the sedimentary basin of Fig. 2. The rock basement subjected to the incidence waves has to be treated as an external problem, whereas the rest of the layers are internal problems. That is to say, a concise formulation of the DBEM (see Eqs. (10) and (11)), based on the geometry of Fig. 2 is:

$\mathbf{H}^{\mathrm{R}} \mathbf{u}_{R}^{\mathrm{t}}=\mathbf{H}^{\mathrm{R}} \mathbf{u}_{R}^{0}+\mathbf{G}^{\mathrm{R}}\left(\mathbf{t}_{R}^{\mathrm{t}}-\mathbf{t}_{R}^{0}\right)$,

$\mathbf{H}^{\mathrm{G}} \mathbf{u}_{G}^{\mathrm{t}}=\mathbf{G}^{\mathrm{G}} \mathbf{t}_{G}^{\mathrm{t}}$

$\mathbf{H}^{\mathrm{A}} \mathbf{u}_{\mathrm{A}}^{\mathrm{t}}=\mathbf{G}^{\mathrm{A}} \mathbf{t}_{\mathrm{A}}^{\mathrm{t}}$.

Superindex $t, 0$ have the same meaning as in previous sections: total and free-field. On the other hand, subindex $j=R, G, \ldots, A$ hint at the different layers that compose the medium.

\section{Testing of the DBEM's formulation developed}

The accuracy of the method has been gauged by testing the seismic responses computed by the DEBM at specific 


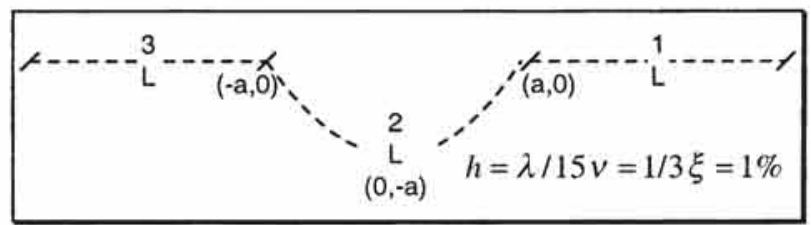

(a)

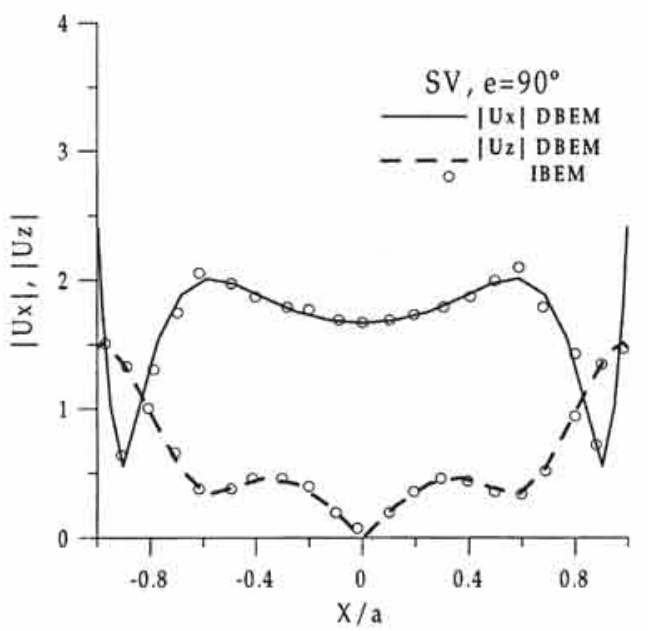

(b)

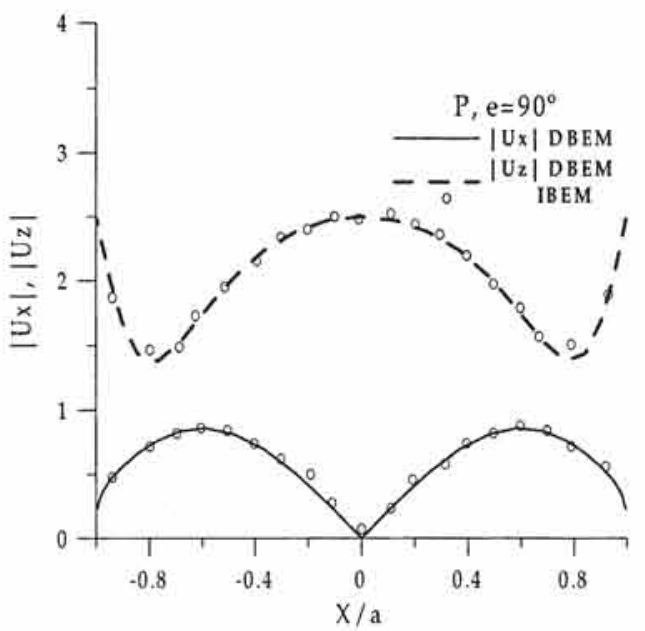

(d)

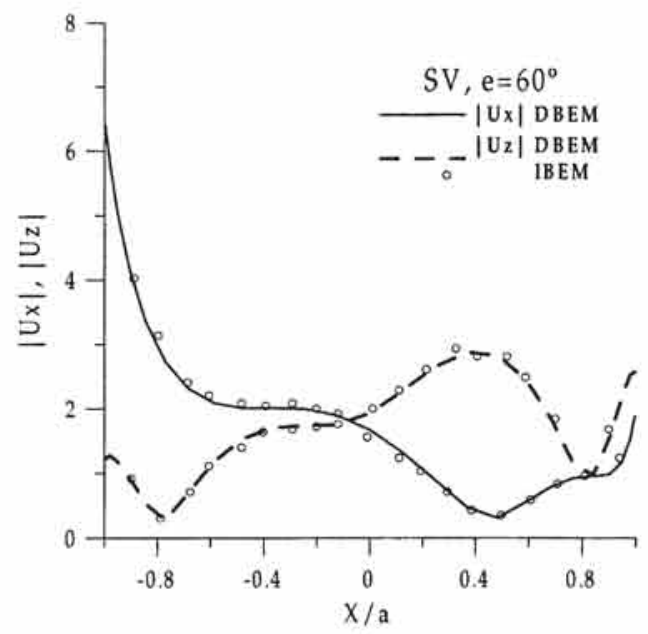

(c)

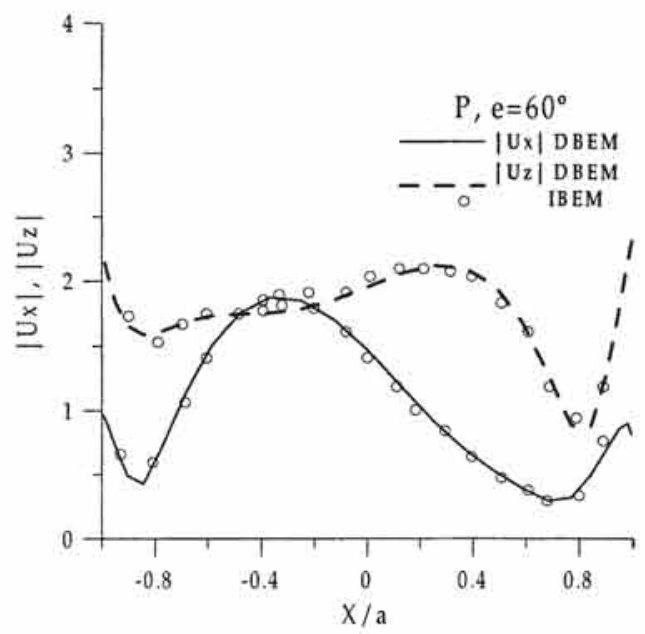

(e)

Fig. 4. Scattering of in-plane waves by a semi-circular canyon. (a) Scheme of the canyon's model of radius $a$. The homogeneous half-space is characterized by Poisson's ratio $1 / 3$, and damping ratio $1 \%$. The element's length of the models's grid is $\lambda / 15$, where $\lambda$ is the S-wavelength. (b)-(e) Comparison of the seismic responses computed on the canyon's surface by the DBEM (lines) with the results of the IBEM (represented by circles). In these figures it is displayed the seismic horizontal $(|\mathrm{Ux}|)$ and vertical ( $|\mathrm{Uz}|$ ) responses for $\mathrm{P}$ and SV waves with emergency angles $e=60^{\circ}$. 90 (measured from the horizontal).

sites, with responses stemmed from the application of other approaches. Specifically these approaches have been: on one hand the numerical technique IBEM developed by Sánchez-Sesma and Campillo [11,12], and on the other a numerical hybrid method presented by Faccioli et al. [22] consisting on the assembling of the FEM and the SEM. These methods have been briefly introduced at the beginning of this work. They have been widely accepted and used in the literature as good techniques for site effect analysis. It should be pointed out that the results presented should not be interpreted as an investigation of the physics of the problem in itself (such type of goal will be addressed in future articles), but merely as a measure of validity of the method developed in this work.

In order to show the validation of the method, it is presented some of the simulations performed for different sites. The first two theoretical models fit two significant site categories in the local effect problem: a semi-circular canyon and semi-elliptical mountain. The third model is also an important site category, but the difference is that it 


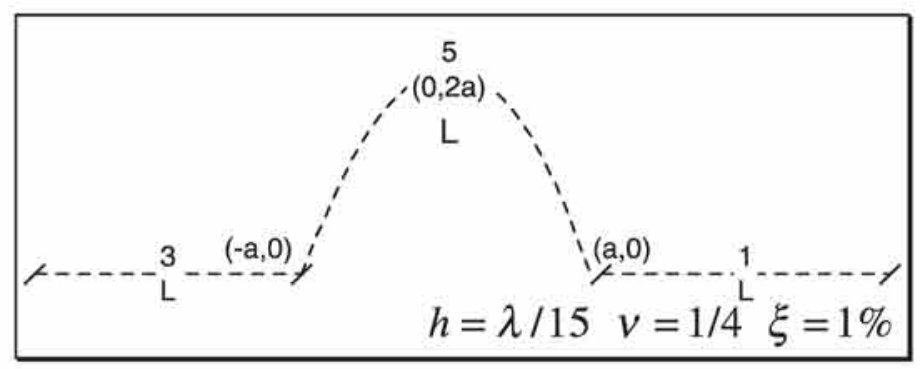

(a)

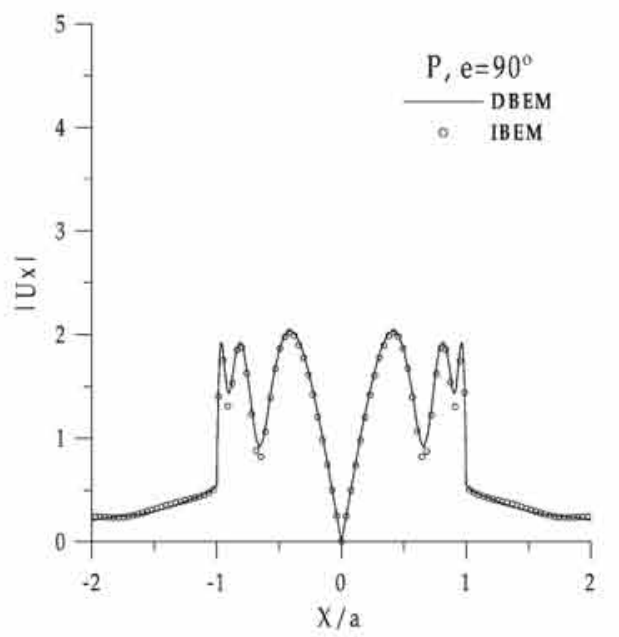

(b)

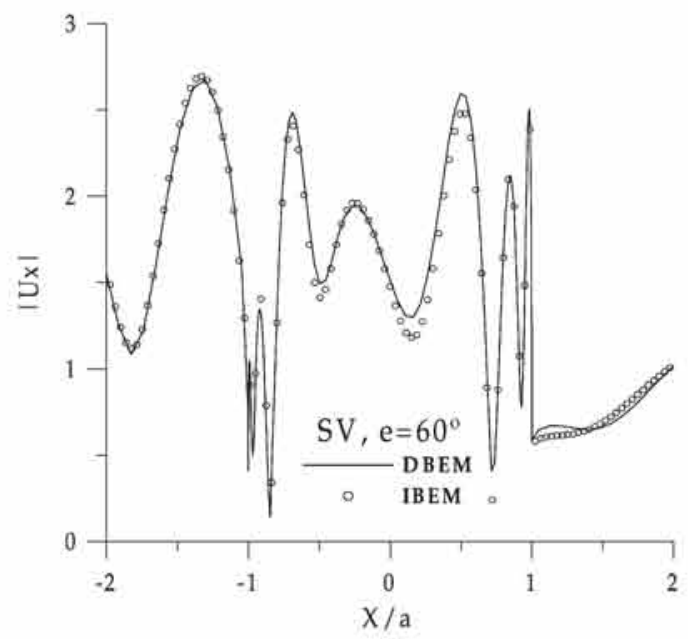

(d)

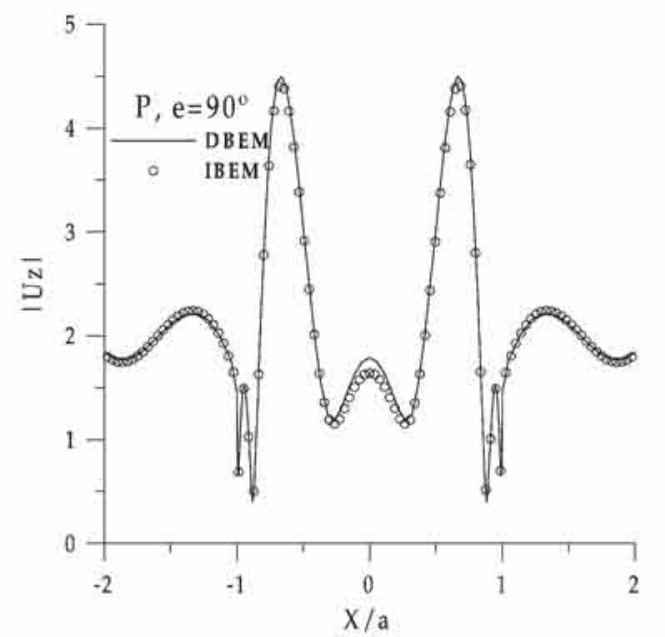

(c)

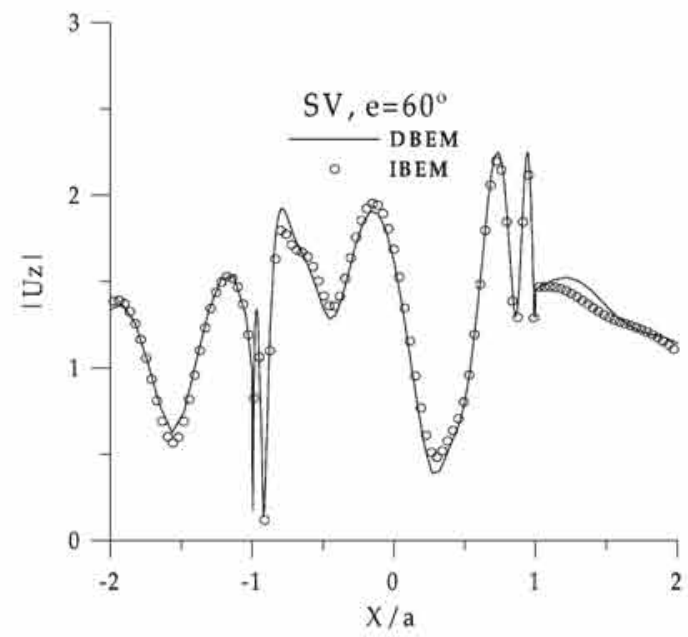

(e)

Fig. 5. Scattering of in-plane waves by a semi-elliptical mountain. (a) Scheme of the elevation's model of height $2 a$. The homogeneous half-space is characterized by Poisson's ratio 1/4, and damping ratio 1\%. The element's length of the models's grid is $\lambda / 15$, where $\lambda$ is the S-wavelength. (b)-(e) Comparison of the seismic responses computed on the mountain's surface by the DBEM (lines) with the results of the IBEM (represented by circles). In these figures it is displayed the seismic horizontal ( $|\mathrm{Ux}|$ ) and vertical ( $|\mathrm{Uz}|$ ) responses for $\mathrm{P}$ and SV waves with emergency angles $e=60^{\circ}, 90^{\circ}$ (measured from the horizontal).

represents a real sedimentary basin. These results are part of a vast study carried out for different models, under diverse radiation characteristics. All results have shown a similar good level of agreement on the testing process between techniques, therefore the DBEM's formulation is validated.

\subsection{Diffraction by a semi-circular canyon and a semi-elliptical mountain}

One of the most extended analysed topographical irregularities in local wave diffraction is the canyon embedded in a homogeneous half-space. Its seismic 


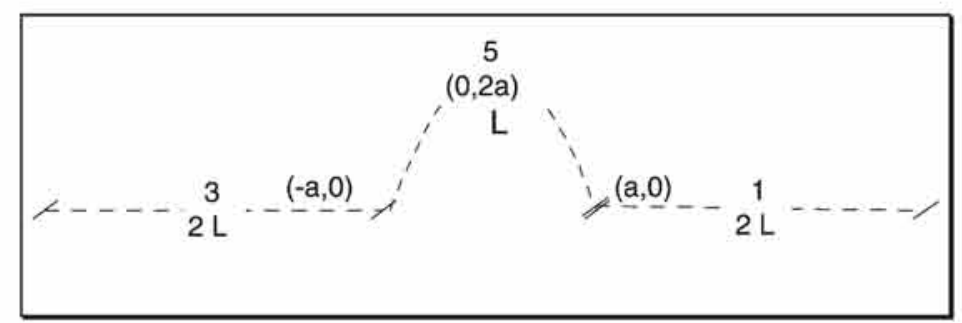

(a)

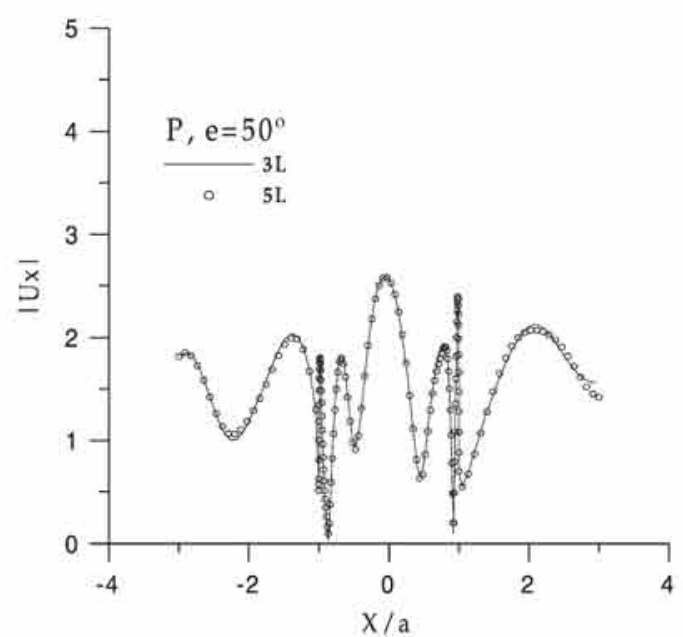

(b)

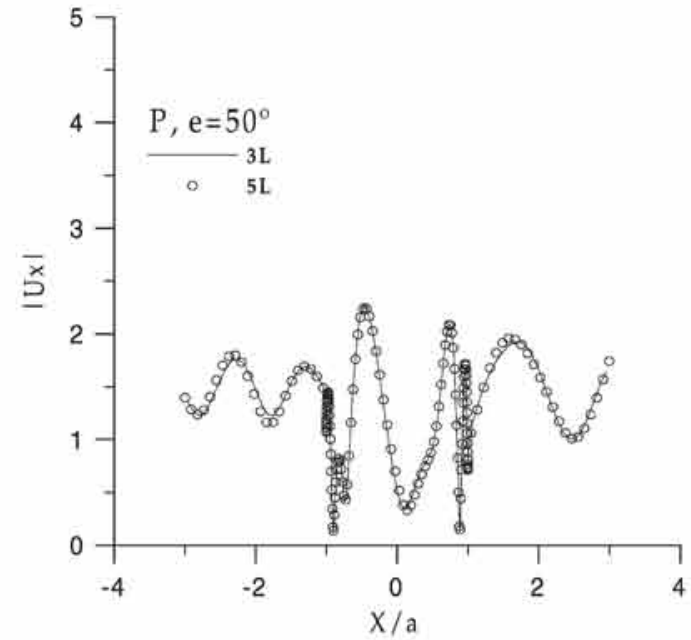

(c)

Fig. 6. (a) In this figure it is shown another mesh corresponding to the semi-elliptical elevation of Fig. 5a. The difference between meshes is the total length $\left(L_{\mathrm{T}}\right)$. (b) and (c) Comparison of the simulations performed by the DBEM on the surface of the semi-elliptical elevation represented on Fig. $5 \mathrm{a}\left(L_{\mathrm{T}}=3 L\right)$ and Fig. $6 \mathrm{a}\left(L_{\mathrm{T}}=5 L\right)$.

response has been assessed by many authors through different techniques: in Wong [27] a generalized inversion technique was applied, Sánchez-Sesma and Campillo [12] developed the IBEM, a 2.5D hybrid formulation, which is an extension of the discrete wavenumber boundary integral equation method, was used by Zhang et al. [28], etc. Therefore this site model has been turned into a site-test for validating new techniques.

Next, the response of an elevated topography such as a semi-elliptical mountain has been computed. This site is a good example to show the validity and ease of the DBEM's scheme-presented in Sections 3.2.1 and 3.2.2-for irregular topographies defined by boundaries outside the homogeneous half-space.

\subsubsection{Frequency-domain response}

In this section a sample of the spectral seismic response computed on the surface of the above mentioned sites is presented. Fig. 4 displays results corresponding to the scattering of $\mathrm{P}$ and $\mathrm{SV}$ waves (amplitude unit of incidence) by a semi-circular canyon of radius $a$, embedded in a homogeneous half-space characterized by Poisson's ratio 1/ 3 , and slightly dissipative with a $1 \%$ hysteretic damping ratio. The seismic response illustrated correspond to the incidence of $\mathrm{P}$ and SV waves with an emergency angle $e=60^{\circ}, 90^{\circ}$ measured from the horizontal. The horizontal $(|\mathrm{Ux}|)$ and vertical ( $|\mathrm{Uz}|)$ Fourier amplitudes computed on the canyon's surface are plotted vs. a normalized $x$ coordinate $(x / a)$. These amplitudes represent the amplification factor due to the irregularity. The dimensionless frequency $\eta=(\omega a / \pi \beta)$, where $\omega$ is the angular frequency and $\beta$ the $S$-wave velocity, is assumed to be equal to 1 . The lines represent the results obtained by the DBEM, while the circles correspond to the IBEM computation. As it can be seen the convergence between results compare favourably both techniques. The discrepancies between them are negligible, they can be discussed in terms of details of the geometry's discretization.

Fig. 5 displays the scattering of $\mathrm{P}$ and $\mathrm{SV}$ waves due to the presence of a semi-elliptical mountain with a maximum height twice the value of its half-width $a$, over a homogeneous half-space characterized by Poisson's ratio $1 / 4$, and slightly dissipative with a $1 \%$ hysteretic damping ratio. The cases illustrated correspond to emergency angles $e=60^{\circ}, 90^{\circ}$, and to a dimensionless frequency $\eta=1.5$. DBEM's and IBEM's results are plotted by lines and circles, respectively. As in the former analysis the agreement found is excellent and therefore the formulation is validated. It has been found discrepancies between the results, that are larger comparing to the ones analysed on the canyon's case, 


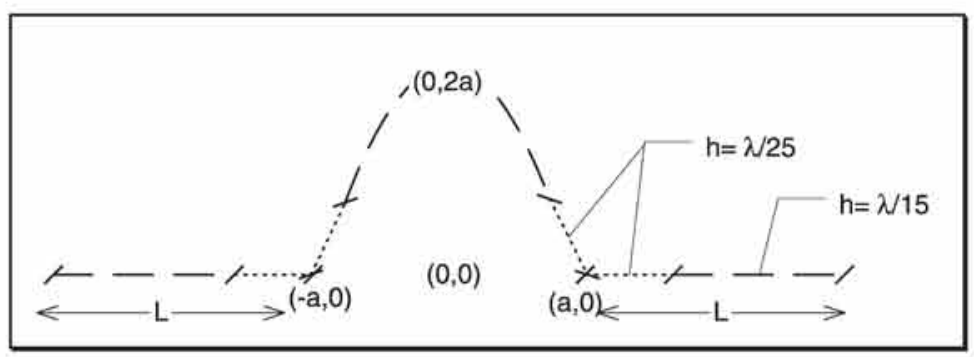

(a)

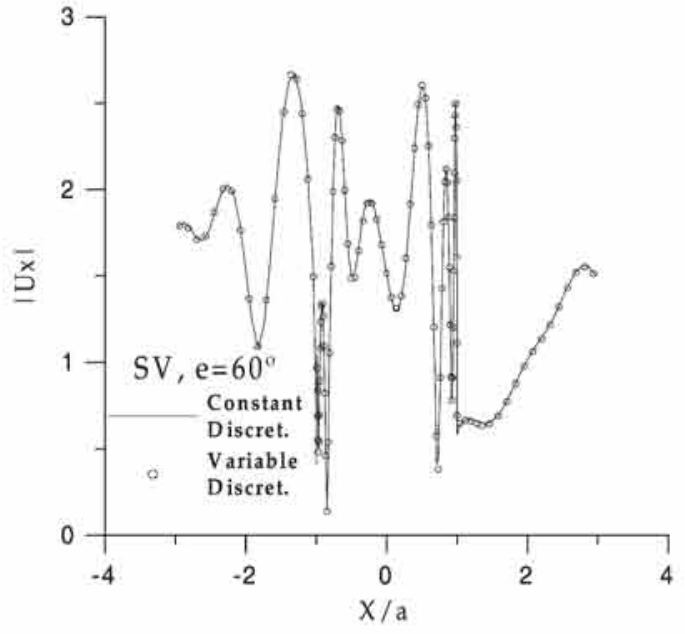

(b)

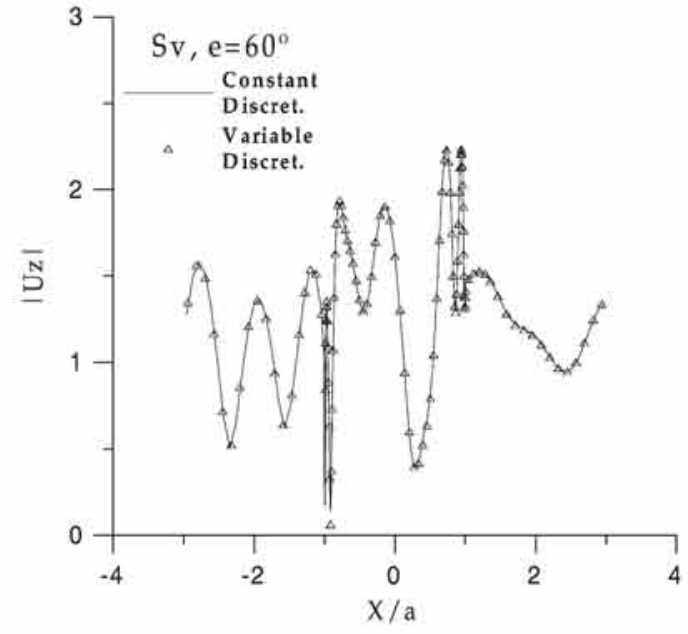

(c)

Fig. 7. (a) The mesh shown in this figure corresponds to the semi-elliptical elevation represented in Fig. 5a. On the contrary to the latter, it is designed with variable discretization (variable element length $h$ ). (b) and (c) Comparison of the simulations performed by the DBEM on the surface of the semi-elliptical elevation represented on Fig. $5 \mathrm{a}(h=\lambda / 15)$ and Fig. $6 \mathrm{a}(h=\lambda / 15, \lambda / 25)$.

although they still remain insignificant as to the validation of the method is concerned. Besides the small details in the discretization applied by both techniques - as it has been already stood out - these differences can also be attributed to the fact that, in the DBEM implementation the input motion, that is the free-field, is produced by incident plane waves in the boundaries within the half-space where the analytical solution is known, whereas the IBEM's free-field is extended to the parts of the topography that are not included in the reference half-space and provides the boundary excitation.

In other engineering fields the method has revealed an important sensitivity to the discretization's criteria adopted, therefore a stability analysis of the method has also been part of the testing procedure. Several grids where designed combining different total discretization lengths $-L_{\mathrm{T}}=3 L$, $5_{L}$ where $L$ is the length of the irregular surface. It has also been taking into account the use of variable discretization along certain parts of the boundary where there is a marked change of its geometry (such as the border of the canyons, valleys, etc.). Element lengths such as $h=\lambda / 15, \lambda / 25$, where $\lambda$ is the $S$ wavelength, have been used. In Figs. 6 and 7 it is shown a sample of the mesh analysis performed. It belongs to the simulations carried out on the semi-elliptical mountain modelled by meshes with different total lengths, see Fig. 6 (incident P-wave $e=50^{\circ}$ ), and variable discretization, see Fig. 7 (incident S-wave $e=60^{\circ}$ ). As it can be seen, it is found a great stability and robustness of the seismic response computed over all the grids analysed. Other analysis have been carried out involving a larger element length $(h=\lambda / 5)$, and excellent agreement has been found between results. In consequence the discretization criteria established, according to computation optimization reasons, is $L_{\mathrm{T}}=3 L$ and $h=\lambda / 5$.

\subsubsection{Time-domain response}

The test of the formulation presented is completed examining the edge effects due to the finite size of the discretized boundary. For this aim, synthetic seismograms were computed using the FFT algorithm for a Ricker wavelet. Time series were obtained from the transfer functions estimated at receivers placed along the free surface. Fig. 8 shows a sample of the synthetic seismograms, computed at the surface of the semi-circular canyon shown on Fig. 4 (Ricker wavelet's central frequency is $f_{\mathrm{p}}=1.5 \beta / 2 a=2.6 \mathrm{~Hz}, \eta=1$ ), for the incidence of $\mathrm{P}$ and SV waves $\left(e=90^{\circ}, 70^{\circ}\right.$, respectively). Fig. 9 shows a sample of the synthetic seismograms, computed at 


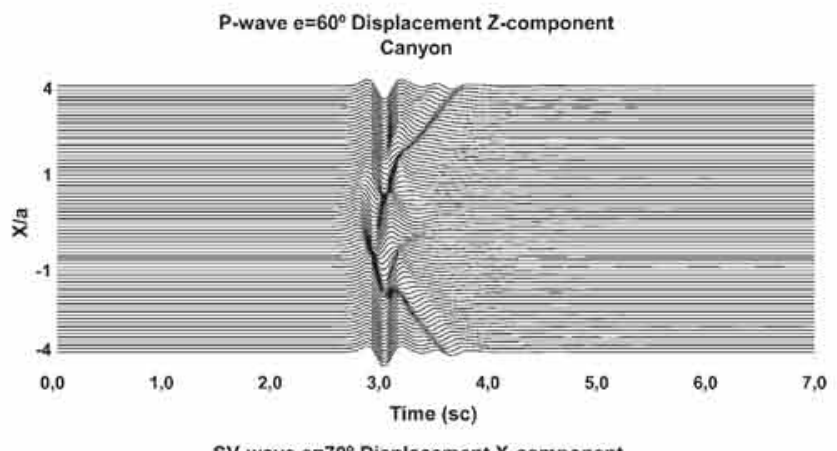

SV-wave $e=70^{\circ}$ Displacement $X$-component

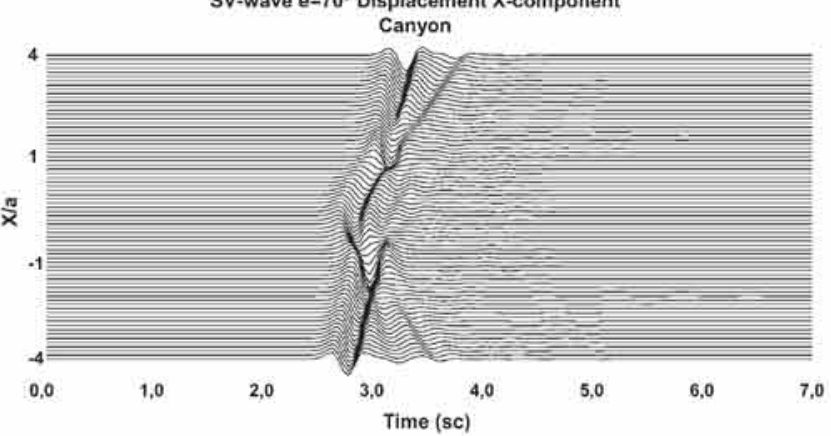

Fig. 8. Time-domain displacement response of a semi-circular canyon (Fig. 4a) to incident $\mathrm{P}\left(e=60^{\circ}\right)$ and SV $\left(e=70^{\circ}\right)$ waves. The incident time signal is a Ricker wavelet with central frequency $f_{\mathrm{p}}=1.5 \beta / 2 a=2.6 \mathrm{~Hz}$. The stations are located along the free surface of the half-space, at a horizontal dimensionless horizontal coordinate $x / a$.

the surface of the semi-elliptical elevation shown on Fig. 5 (Ricker wavelet's central frequency is $f_{\mathrm{p}}=5.4 \mathrm{~Hz}, \eta=1.5$ ), for the incidence of $\mathrm{P}$ and SV waves $\left(e=90^{\circ}, 60^{\circ}\right.$, respectively). As it can be seen, the nonphysical waves are negligible on all the examples shown, therefore the seismic response is not influenced by the truncation of the free surface. This result, together with the other aspects already discussed, confirms the efficiency of the DBEM's formulation on site effects estimation.

The time response also contributes to estimate the range of the effects caused by the local conditions. Although as it has been already argued, this deeper analysis of the diffraction phenomena is beyond the scope of this work, synthetics can be taking as an example of the complicated interference patterns that can take place. At a first sight it can be outlined significant features on the seismic response such as: the existence of diffracted waves at the edge of the irregularities $(x / a=-1,1)$, the creeping waves along the irregularity surface, that induce important variations of the ground motion at and around the irregularity.

\subsection{Diffraction by a multilayer sedimentary basin}

The extension of the DBEM's formulation developed, as to the complex sites is concerned, is being validated by the computation of the seismic response at a real site such as the Volvi sedimentary basin. In recent years, this basin

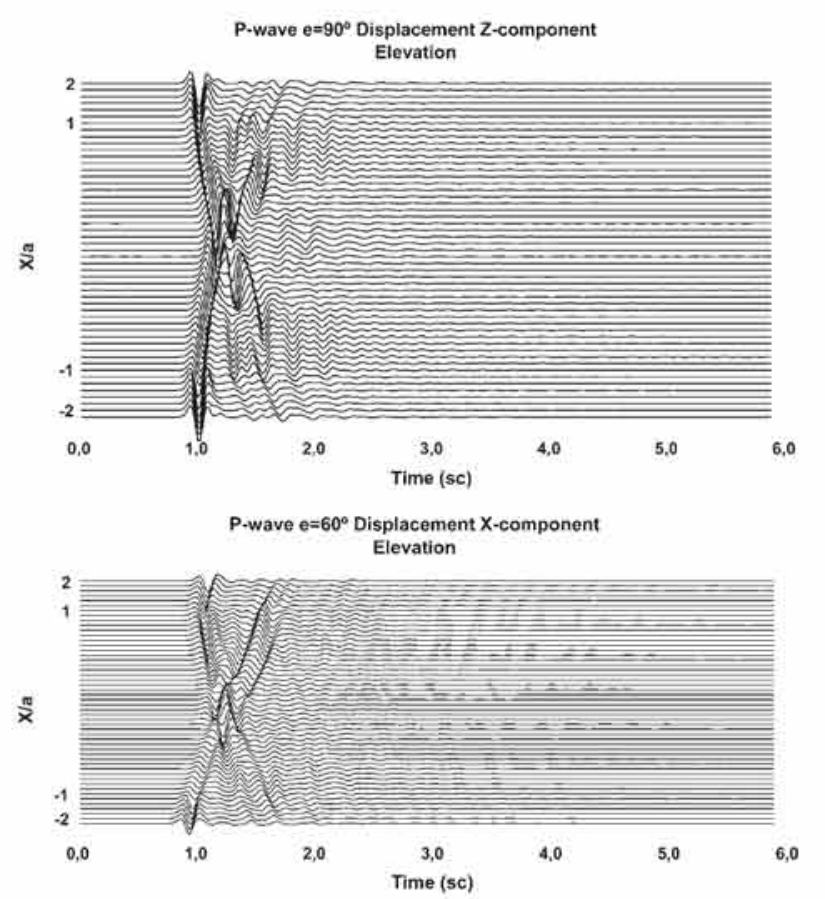

Fig. 9. Time-domain displacement response of a semi-elliptical elevation (Fig. 5a) to incident $\mathrm{P}\left(e=90^{\circ}\right)$ and SV $\left(e=60^{\circ}\right)$ waves. The incident time signal is a Ricker wavelet with central frequency $f_{\mathrm{p}}=5.4 \mathrm{~Hz}$. The stations are located along the free surface of the half-space, at a horizontal dimensionless coordinate $x / a$.

has become a test-site for local site effect studies. It is due to the detailed information available on the subsoil structure, and the existence of a large recorded database of small and intermediate intensity earthquakes. There is a vast literature published, dedicated to this basin with different purposes $[22,26,29-33]$. Consequently in this section the description's site is limited to locate the basin, as well as, to propose a model based on the published geometry and dynamic properties of soil deposits. As it has already been introduced, the DBEM's

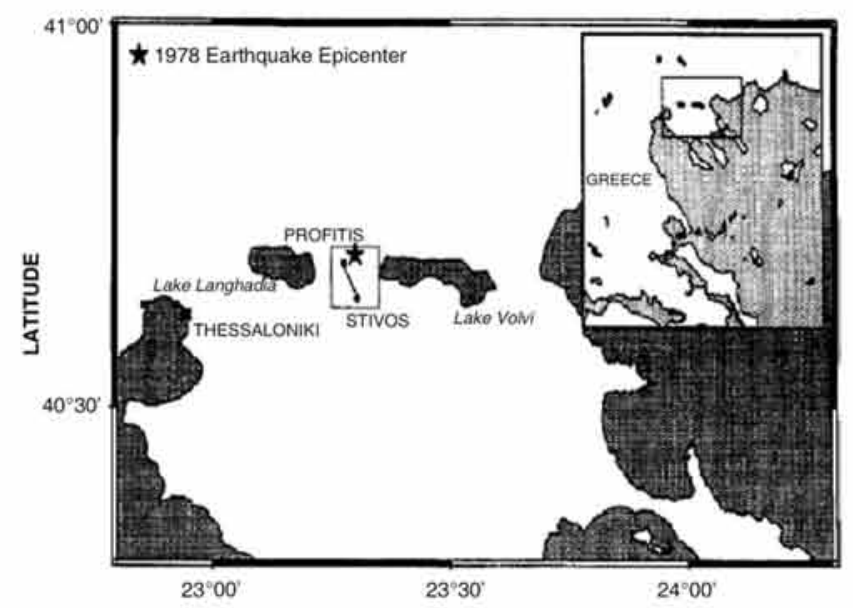

Fig. 10. Map of the localization of the Volvi sedimentary basin. The NNWSSE 2D soil structure of the basin depicted in Fig. 2, is represented, in this figure, by the line that joins Profitis (PRO) site to Stivos (STE) site. 
Table 1

Properties of the materials that compose the eighth layers of the basin shown in Fig. 2

\begin{tabular}{lllllr}
\hline Layer & $\rho\left(\mathrm{kg} / \mathrm{m}^{3}\right)$ & $\beta(\mathrm{m} / \mathrm{s})$ & Poisson's ratio & $\alpha(\mathrm{m} / \mathrm{s})$ & $Q_{s}{ }^{a}$ \\
\hline$A$ & 1700 & 130 & 0.408 & 330 & 40 \\
$B$ & 1800 & 200 & 0.375 & 450 & 20 \\
$C$ & 1800 & 300 & 0.290 & 550 & 30 \\
$D$ & 2100 & 450 & 0.285 & 820 & 25 \\
$E$ & 2150 & 650 & 0.285 & 1185 & 50 \\
$F$ & 2200 & 900 & 0.285 & 1640 & 60 \\
$G$ & 2500 & 1250 & 0.280 & 2260 & 100 \\
$R$ & 2600 & 2500 & 0.277 & 4500 & 200 \\
\hline
\end{tabular}

a $Q$ value at the reference frequency of $1 \mathrm{~Hz}$.

computation will be contrasted with results achieved by Faccioli et al. [22], by the use of a hybrid pseudospectral approach, consisting on the computation of the FEM together with the SEM.

As it is shown in Fig. 10 the Volvi basin-a site of $5.5 \mathrm{~km}$ wide and $200 \mathrm{~m}$ deep-is located between the Langhada and Volvi lakes in the Mygdonian graben, $30 \mathrm{~km}$ to the east of Thessaloniki in northern Greece. The DBEM's computations have been carried out over a mesh which is based on the digitization of the 2D valley's structure analysed by Faccioli et al. [22]. In Fig. 2 and Table 1 are shown a cross-section of this structure-denoted from now on as model $I$-and the soil properties, respectively. Because of time and memory requirements-which are at present being optimized for further studies in heavy situations-the mesh designed in this work, model II-has been simplified. Fig. 11 shows the structure of model II analysed. As it is shown, the difference between both

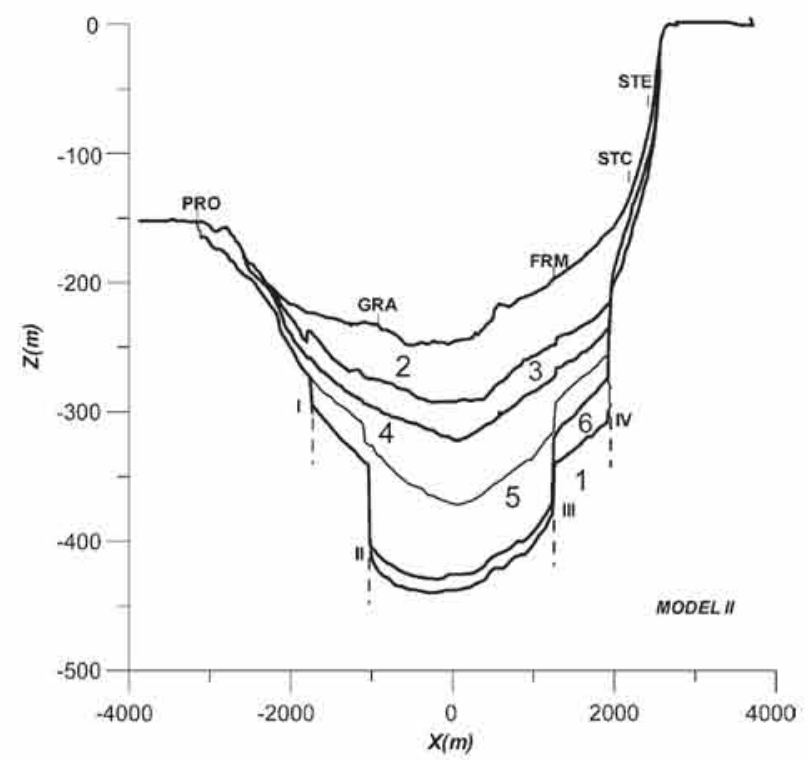

Fig. 11. Structure of the Volvi sedimentary basin analysed in this work and denoted by model II. It has been obtained by the digitation of model I in Fig. 2.
Table 2

Properties of the materials that compose the six layers of the basin shown in Fig. 11

\begin{tabular}{llllrr}
\hline Layer & $\rho\left(\mathrm{Kg} / \mathrm{m}^{3}\right)$ & $\beta(\mathrm{m} / \mathrm{s})$ & $v$ & $\alpha(\mathrm{m} / \mathrm{s})$ & $Q_{\mathrm{S}}{ }^{\mathrm{a}}$ \\
\hline 1 & 2600 & 2500 & 0.277 & 4500 & 200 \\
2 & 1800 & 250 & 0.332 & 498 & 25 \\
3 & 2100 & 450 & 0.285 & 820 & 25 \\
4 & 2150 & 650 & 0.285 & 1185 & 50 \\
5 & 2200 & 900 & 0.285 & 1640 & 60 \\
6 & 2500 & 1250 & 0.280 & 4500 & 100 \\
\hline \multirow{2}{*}{${ }^{*} Q_{\text {S }}$ value at the reference frequency $1 \mathrm{~Hz}}$.
\end{tabular}

models comes down to the merging of layers $\mathrm{A}-\mathrm{C}$ (model I) into layer 2 (model II). In Table 2 is shown the soil properties of the last. The material properties assigned to layer 2 (model II) correspond to the mean values of layers A-C (model I). The comparison between techniques will be performed over the seismic responses calculated on the five sites located on both models: PRO, GRA, FRM, STC, STE. These have been approximately located during the digitization process of model I.

The simulations carried out correspond to the vertical incidence of a SV wave (amplitude unit) on the rock basement (layer 1). In Fig. 12 the horizontal transfer functions calculated with the DBEM at the above mentioned stations (column a) are plotted vs. the ones published by Faccioli et al. [22] (column b). As it can be seen, both responses coincide in their main features: amplification levels, resonance frequencies. It can be concluded that DBEM's calculated response is consistent with the results presented by Faccioli et al.

In a closer analysis of the results, it has to be outlined that there have been found larger discrepancies between the transfer functions obtained by both techniques in stations FRM and GRA, than in the rest of the stations. This difference can be attributed to the fact that, FRM and GRA sites are located where the local superficial conditions differ more between both models, I and II. It also has to be pointed out that both stations are strategically situated over two vertical faults where, in a deeper analysis that has been carried out and which will be presented in a future work, is shown that, there is a great variability of the soil response, and any uncertainty in the location process may induce to differences on the transfer functions simulated by both methods.

\section{Conclusions}

It has been presented the adaptation of the formulation of DBEM, for computing 2D site effects due to the diffraction of in-plane waves at irregular sub and superficial laterally varying layered media. The method works directly on frequency-domain and uses the exact full-space time- 

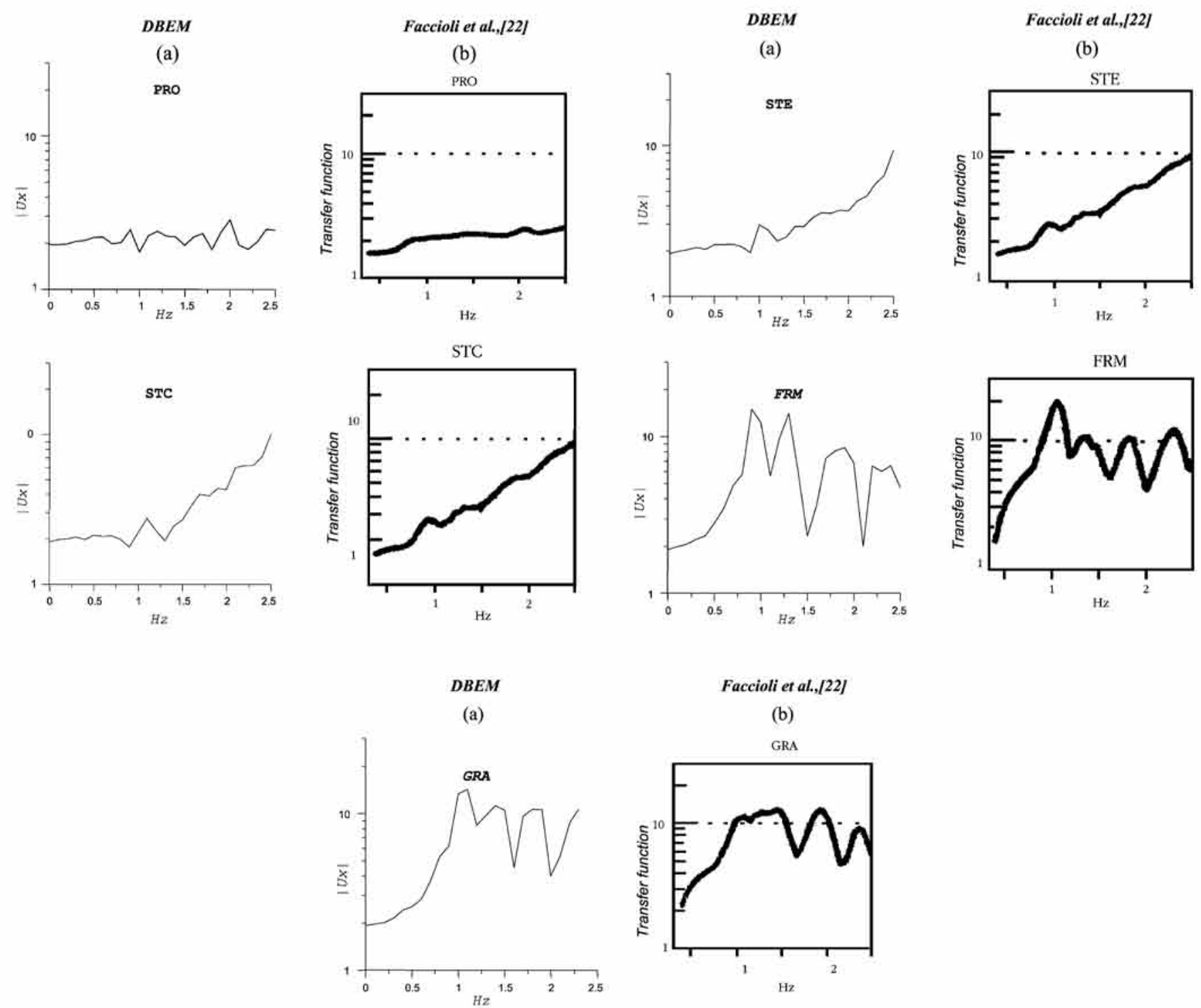

Fig. 12. Comparison between the horizontal transfer functions computed by the DBEM at different sites of model II (Fig. 11), and the results obtained by Faccioli et al. [22] at the same sites in model I (Fig. 2).

harmonic fundamental solution. The formulation is implemented in such a way that it permits the systematic simulation of transfer functions for a certain spectral range at any specific site in the regions.

The validation process has been carried out by comparison of the seismic response computed by the DBEM on theoretical and real soil models, with those obtained by the application of other numerical techniques. As results of the tests attained, there has been found an excellent agreement between the techniques. Thus, the DBEM's formulation presented in this work is validated for seismic responses on complex local conditions. It stands out the straight-forwardness of the approach to directly obtain the dynamical regime - displacements and tractions - with no other intermediate source of error, rather than the ones stemmed from its own approximate character. The versa- tility of the method is partly due to its resolution based on the fulfillment of continuity conditions of displacements and tractions, at boundaries between materials. It is also remarkable that the formulation is developed with no need of analytical extensions of the free-field beyond the half-space, since it is evaluated at boundaries completely embedded in it.

On the other hand, the method has showed a high level of stability and robustness, which has not been found in former applications of the method for other engineering purposes. Therefore it suffices to discretize the site model according to the criteria which optimizes the computing time and memory requirements: shortest total surface length, $L_{\mathrm{T}}=3 L$ ( $L$ is the length of the irregular surface), and the maximum constant element size used $h=\lambda / 5$. As it has been shown in the real soil model analysis 
performed (the Volvi sedimentary basin), these criteria enables to undertake the estimation of the seismic responses at real sites characterized by large dimensions and complex media. Nevertheless the application of the method in terms of seismic risk analysis-where the high frequency information is important-requires an optimization of the resolution of the final system of equations. This issue is at present being undertaking, and it will provide the extension of the DBEM's formulation to the 3D case.

\section{Acknowledgements}

Thanks are given to Rossana Vai from the Instituto Mexicano del Petroleo in México, for her essential contribution to the development of this work. We thank Belén Benito and Esther Jimenez from the Escuela Universitaria de Ingeniería Técnica Topográfica of the Universidad Politécnica de Madrid, for their helpful comments and support to this work. We are also most grateful to José Domínguez from the Escuela Superior de Ingenieros of the Universidad de Sevilla, for his technical advices.

\section{References}

[1] Aki K. Local site effects on strong ground motion in earthquake engineering. Soil dynamics II, recent advances in ground motion evaluation. Am Soc Civil Engng/Geotech Spec Publ 1988; 20:103-55.

[2] Geli L, Bard P-Y, Jullien B. The effect of topography on earthquake ground motion: a review and new results. Bull Seismol Soc Am 1988; 78:42-63.

[3] Bard P-Y. Effects of surface geology on ground motion: recent results and remaining issues. In: Proceedings of the 10th European Conference on Earthquake Engineering, Vienna, Austria; 1995

[4] Sánchez-Sesma FJ, Benites R, Bielak J. The assessment of strong ground motion. What lies ahead? In: 11th World Conference on Earthquake Engineering, Acapulco, México; 1996

[5] Bard P-Y. Site effects and seismic microzonation: recent advances, knowledge gaps and perspectives for applications. In: Sixth Forum of Seismic Zonation, Ibero-Magrebian Region Conferenca. Barcelona, Spain; 1996

[6] Álvarez-Rubio S. El Efecto Local sobre el Movimiento Sísmico del Suelo: Fenomenología y Resultados Recientes. In: Física de la Tierra, vol. 11. España: Universidad Complutense de Madrid; 1999. p. 14173

[7] Sánchez-Sesma FJ. Strong ground motion and site effects. In: De Beskos DE, Anagnostopoulos SA, editors. Computer analysis and design of earthquake resistant structures. Southampton: Computational mechanics publications; 1996. p. 201-39. (BAS) [chapter 4].

[8] Brebbia CA, Progress in boundary element methods, vol. 1. Plymouth: Pentech Press; 1981.

[9] Luco JE, Wong HL, De Barros FCP. Three-dimensional response of a cylindrical canyon in a layered half-space. Earthquake Engng Struct Dyn 1990;799-817.

[10] Luco JE, De Barros FCP. On the three-dimensional seismic response of a class of cylindrical inclusions embedded in layered media. In:
Proceedings of the Sixth International Conference of Soil Dynamics and Earthquake. Bath, UK; 1993. p. 565-80

[11] Sánchez-Sesma FJ, Campillo M. Diffraction of P, SV, and Rayleigh waves by topographic features: a boundary integral formulation. Bull Seismol Soc Am 1991;81:2234 53 .

[12] Sánchez-Sesma FJ, Campillo M. Topographic effects for incident $P$, SV and Rayleigh waves. Tectonophysics 1993;218:113-25.

[13] Pedersen H, Sánchez-Sesma FJ, Campillo M. Three-dimensional response of two-dimensional topographies. Bull Seismol Soc Am 1994;84:1169-83.

[14] Sánchez-Sesma FJ, Luzón F. Seismic response of three-dimensional alluvial valleys for incident $\mathrm{P}, \mathrm{S}$ and Rayleigh waves. Bull Seismol Soc Am 1995;85:269-84.

[15] Luzón F, Sánchez-Sesma FJ, Rodríguez-Zúñiga IL, Posadas AM, García JM, Martín J, Romacho MD, Navarro M. Diffraction of P, S and Rayleigh waves by three-dimensional topographies. GIJ 1997; 129:571-8.

[16] Kawase H. Time-domain response of a semicircular canyon for incident SV, P and Rayleigh waves calculated by the discrete wavenumber boundary element method. Bull Seismol Soc Am 1988; 78:1415-37.

[17] Kim J, Papageorgiou AS. Discrete wavenumber boundary-element method for 3-D scattering problems. J Engng Mech ASCE 1993; 119(3):603-24.

[18] Papageorgiou AS, Pei D. A discrete wavenumber boundary element method for study of the 3-D response of 2-D scatterers. Earthquake Engng Struct Dyn 1998;27:619-38.

[19] Reinoso E, Wrobel LC, Power H. Preliminary results of the modelling of the Mexico City valley with two-dimensional boundary element method for the scattering of SH waves. Soil Dyn Earthquake Engng 1994;12:457-68.

[20] Reinoso E. Boundary element modelling of scattering from topographical structures with applications to the Mexico City valley. $\mathrm{PhD}$ Thesis, Wessex Institute of Technology. University of Portsmouth, England; 1994

[21] Domínguez J. Boundary elements in dynamics. Southampton/ Amsterdam: Computational Mechanics Publications/Elsevier, 1993.

[22] Faccioli E, Paolucci R, Vanini M. TRISEE 3D site effects and soilfoundation interaction in earthquake and vibration risk evaluation. In: European Commission, Directorate General XII for Science, Research and Development. 1994-1998 Environments and Climate Programme-Climate and Natural Hazards Unit

[23] Tadeu A, Kausel E. Green's functions for two-and-a-half dimensional elastodynamic problems. J Engng Mech ASCE 2000;126(10): 1093-7.

[24] Tadeu A, António J. 2.5 Green's functions for elastodynamic problems in layered acoustic and elastic formations. J Comput Model Engng Sci CMES 2001:2(4):477-95

[25] Ortiz-Alemán C, Sánchez-Sesma FJ, Rodriguez-Zúñiga JL, Luzón F. Computing topographical 3-D site effects using a fast IBEM/ conjugate gradient approach. Bull Seismol Soc Am 1998;88(2): 393-9.

[26] Álvarez-Rubio S. Aplicación del Método de Elementos de Contorno a la Cuantificación del Efecto Local en el Registro Sísmico. PhD Thesis, Escuela Técnica Superios de Ingenieros Industriales, Universidad Politécnica de Madrid, Spain; 2001

[27] Wong HL. Effect of surface topography on the diffraction of P, SV, and Rayleigh waves. Bull Seismol Soc Am 1982;72:1167-83.

[28] Zhang B, Papargeorgiou AS, Tassoulas JL. A hybrid numerical technique, combining the finite-element and boundary-element methods, for modelling the $3 \mathrm{D}$ response of $2 \mathrm{D}$ scatterers. Bull Seismol Soc Am 1998;88:1036-50.

[29] Jongmans D, Pitilakis K, Demanet D, Raptakis D, Riepl J, Horrent C, Tsokas G, Lontzetidis K, Bard PY. EURO-SEISTEST: determination of the geological structure of the Volvi basin and validation of the basin response. Bull Seismol Soc Am 1998;88: $473-87$. 
[30] Raptakis D, Chávez-García FJ, Makra K, Pitilakis K. Site effects at euroseistest. I. Determination of the valley structure and confrontation of observations with 1D analysis. Soil Dyn Earthquake Engng 2000; 19(1):1-22.

[31] Chávez-García FJ, Raptakis D, Makra K, Pitilakis K. Site effects at euroseistest. II. Results from 2D numerical modeling and comparison with observations. Soil Dyn Earthquake Engng 2000; $19(1): 23-40$.
[32] Makra K, Raptakis D, Chávez-García J, Pitilakis K. Site effects and design provisions: the case of euroseistest. Pure Appl Geophys 2001; 158:2349-67.

[33] Marrara F, Suhaldoc P. 2-D modelling of site effects along the EUROSEISTEST array (Volvi Graben Greece). Pure Appl Geophys 2001; 158:2369-88.

[34] París F, Cañas J. Boundary element method. Fundamentals and applications. Oxford: Oxford University Press; 1997. 